\title{
Germanium-on-silicon platforms for nonlinear photonics in the mid-infrared
}

\author{
A. C. Peacock*, L. Shen, N. Healy, C. J. Mitchell, J. S. Penades, M. Nedeljkovic, and G. Z. Mashanovich \\ Optoelectronics Research Centre \\ University of Southampton \\ Southampton SO17 1BJ, United Kingdom \\ acp@orc.soton.ac.uk
}

\begin{abstract}
We review our progress in the characterization of the nonlinear transmission properties of low loss germanium-onsilicon waveguides. Simple pump-probe experiments are employed to demonstrate their use for all-optical control. Keywords-Group IV Photonics; Nonlinear Optics; All-Optical Devices.
\end{abstract}

\section{INTRODUCTION}

Group IV mid-infrared photonics is currently attracting considerable attention for applications in sensing and spectroscopy as this wavelength region contains strong vibrational signatures for a number of gases and molecules. Although much of the work in this area has leveraged the welldeveloped silicon-on-insulator (SOI) platform, more recently several demonstrations of low loss germanium-on-silicon $(\mathrm{Ge}-$ on-Si) waveguides have emerged $[1,2,3]$. As with their silicon counterparts, the germanium waveguides can be fabricated from CMOS compatible platforms using standard lithography and etch methods, thus facilitating integration with other photonic chip-based technologies [4]. Here we review our progress in characterizing the nonlinear transmission properties of the Ge-on-Si rib waveguides. By exploiting the large nonlinear parameters across the $2-4 \mu \mathrm{m}$ regime, we have demonstrated their use for ultrafast all-optical control.

\section{FABRICATION AND CHARACTERIZATION}

The Ge-on-Si waveguides were fabricated from a $2 \mu \mathrm{m}$ thick germanium layer that was epitaxially deposited on a silicon substrate. The rib structures were defined using lithographic patterning and reactive-ion etching methods to have an etch depth of $1.2 \mu \mathrm{m}$ and a core width of $2.25 \mu \mathrm{m}$. To help with the coupling, input and output tapers were fabricated at each end with a maximum width of $10 \mu \mathrm{m}$ and a flare angle that was carefully chosen to minimize coupling losses across the investigated wavelength range. Waveguides of different lengths were incorporated on the chip by introducing four identical bends, of varying separation, in each core section. The inset in Fig. 1 shows a SEM cross-sectional image of a typical input facet showing that the ribs were well formed.

To characterize the optical transmission properties of the waveguides, linear loss measurements were performed using various mid-infrared sources over the wavelength range 1.9$3.8 \mu \mathrm{m}$. An effective cut-back method was applied by comparing the transmitted power through waveguides of different lengths. A summary of the loss measurements taken over the entire wavelength range is provided in Fig. 1[5]. The high loss value recorded at $1.95 \mu \mathrm{m}$ is expected as it is close to germanium's band edge, and beyond $2 \mu \mathrm{m}$ the losses flatten out to a consistently low value of $\sim 3 \mu \mathrm{m}$. The results indicate that the Ge-on-Si waveguides should exhibit a broad transmission window across much of the mid-infrared regime.

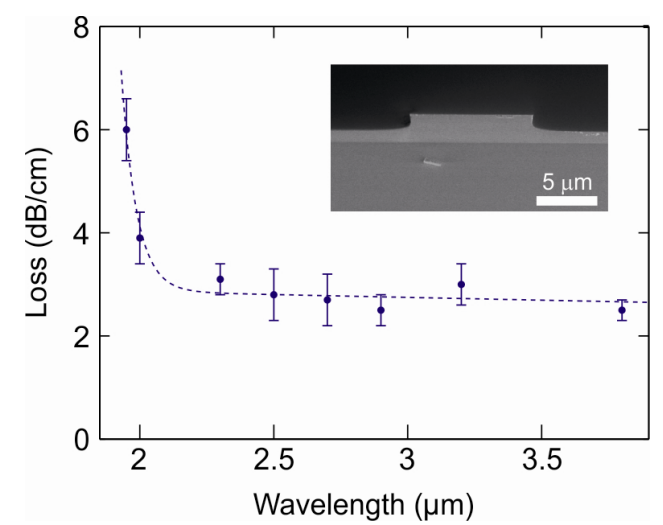

Fig. 1 Linear losses over the 1.9-3.8 $\mu \mathrm{m}$ wavelength range. Inset: SEM cross-sectional image of an input facet.

\section{NONLINEAR ABSORPTION CHARACTERIZATION}

Our starting point for benchmarking the nonlinear performance of the Ge-on-Si waveguides begins with characterization of the two-photon absorption (TPA). Although several measurements of the TPA parameter $\beta_{\mathrm{TPA}}$ have been conducted in bulk Ge samples, no attempt has been made to check these against measurements of the epitaxially deposited waveguide material. To undertake this task we have performed simple high power transmission experiments, where the pump pulses are delivered from a high power optical parametric oscillator (OPO) tunable over the entire TPA window 1.9-3.7 $\mu \mathrm{m}$ [6]. Fig. 2(a) plots the results of the measurements for selected pump wavelengths. For all wavelengths up to $3.4 \mu \mathrm{m}$, it is obvious that the output powers saturate due to the strong nonlinear absorption caused by TPA. In contrast, the largely linear trend exhibited for transmission at $3.7 \mu \mathrm{m}$, indicates that TPA is essentially negligible at this wavelength. Fitting the data with equations for the high power propagation (solid curves), we obtain the values for $\beta_{\mathrm{TPA}}$ presented in Fig. 2(b). The trend of decreasing $\beta_{\mathrm{TPA}}$ is as we would expect across this regime, and the high recorded values $(1000 \times$ larger than 

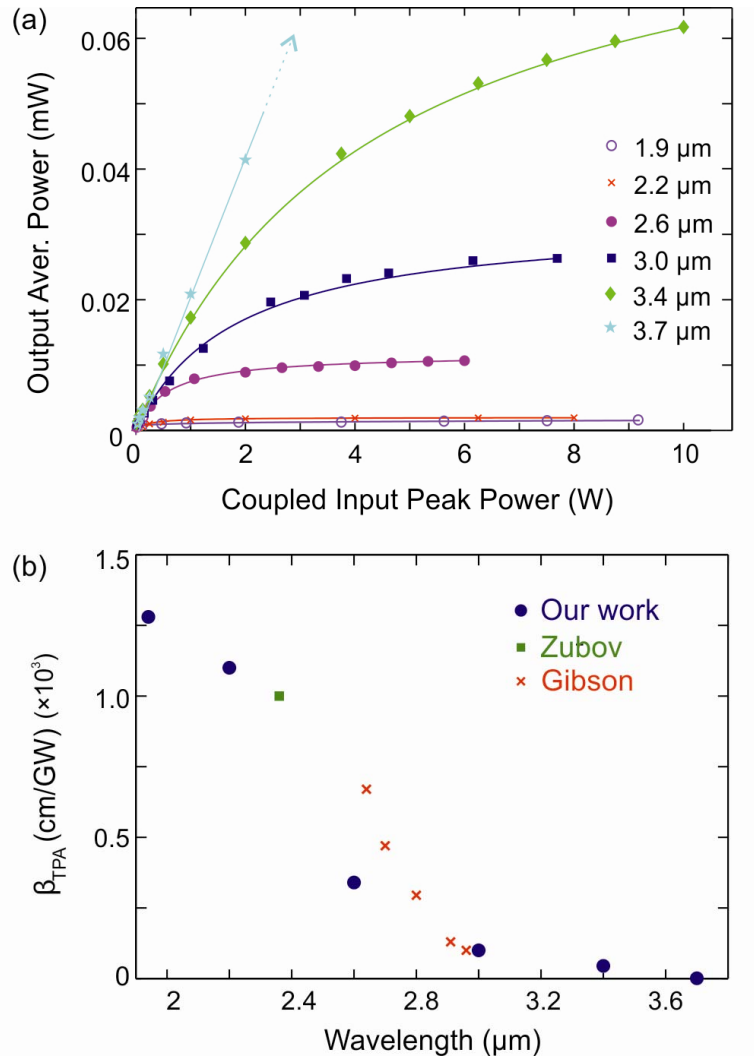

Fig. 2 (a) Nonlinear absorption measurements in the Ge-on-Si waveguides, together with numerical fitted curves (solid lines). (b) Measured TPA parameters as a function of wavelength, together with data points obtained from previous bulk measurements, as labeled.

silicon) are in good agreement with those obtained for bulk germanium by Zubov et al. [7] and Gibson et al. [8].

\section{All-Optical Modulation}

Although such high nonlinear absorption is undesirable in many applications, the ultrafast nature of TPA can be exploited to realize a number of all-optical processing functions such as pulse shapers, logic gates, modulators and switches [9]. Here we make use of the large $\beta_{\mathrm{TPA}}$ parameter at $\sim 2 \mu \mathrm{m}$ to demonstrate a high-speed, all-optical cross-absorption modulation (XAM) process using a simple pump-probe experiment. As this scheme is based on TPA, one photon from the high intensity pump is combined with another from the weak probe so that there is enough energy to span the bandgap and be absorbed. As a result, photons will be removed from the probe that is otherwise too weak to induce TPA, producing a modulation that is an inversion of the pump. To implement the pump-probe configuration, we split a high power mode-locked fiber laser operating at $1.95 \mu \mathrm{m}$ into the pump and probe elements. The two pulses are then recombined inside the Geon-Si waveguide as a function of delay. Fig. 3 plots the absorption of the weak probe as a function of delay for a coupled pump peak power of $10 \mathrm{~W}$. The measured response clearly shows that the ultrafast absorption due to TPA occurs on the timescale of the pump pulse $\left(\mathrm{T}_{\mathrm{fwhm}} \sim 5 \mathrm{ps}\right)$. Significantly, we believe that the extinction ratio measured here of $8.1 \mathrm{~dB}$ is

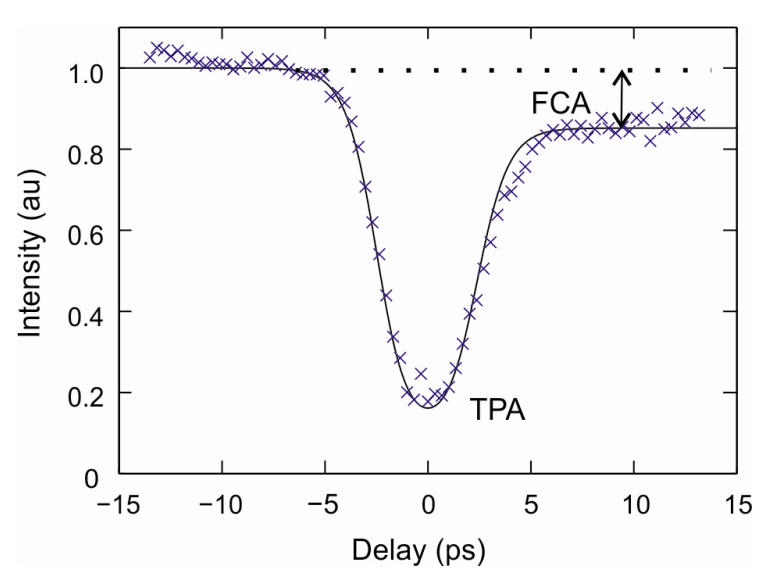

Fig. 3 High speed nonlinear absorption (crosses) of a weak probe, together with the simulated fit (solid line).

the highest reported in any of the group IV waveguides to date, which we attribute to the very large nonlinear absorption accessed in this experiment. Thus as $\beta_{\mathrm{TPA}}$ remains strong across the $2-3 \mu \mathrm{m}$ range, it should be straightforward to extend this scheme to develop high-speed, high-extinction modulators across this important wavelength region.

\section{CONCLUSION}

We have made the first step towards characterizing the nonlinear transmission properties of the Ge-on-Si waveguides by determining the TPA parameter across the 1.9-3.7 $\mu \mathrm{m}$ transparency window and demonstrating its use for efficient all-optical modulation. Ongoing work to characterize the nonlinear refractive index will be discussed, through which we will continue to benchmark the nonlinear performance of this new class of waveguide. Our results to date indicate that the Ge-on-Si waveguides are a viable platform for the development of nonlinear optical devices in the mid-infrared.

\section{ACKNOWLEDGMENT}

The authors acknowledge EPSRC for financial support under the project MIGRATION. G. Z. M. thanks the Royal Society for his University Research Fellowship.

\section{REFERENCES}

[1] Y. Chang et al., Opt. Lett. vol. 37, pp.2883, 2012.

[2] A. Malik et al., Appl. Phys. Lett. vol. 103, 161119, 2013.

[3] M. Nedeljkovic et al., Photon. Technol. Lett. vol. 27, pp.1040, 2015.

[4] G. Roelkens et al., Opt. Mater. Express vol. 3, pp.1523, 2013.

[5] L. Shen et al., Opt. Lett. vol. 40, pp.268, 2015.

[6] L. Shen et al., Opt. Lett. vol. 40, pp.2213, 2015.

[7] B. V. Zubov et al., JETP Letters vol. 9, pp.130, 1969.

[8] A. F. Gibson et al., J. Phys. C vol. 9, pp.3259, 1976.

[9] X. Sang et al., J. Optoelecton. Adv. M. vol.11, pp.15, 2008. 